\title{
Criminologie
}

\section{Le droit aux soins de santé mentale en milieu carcéral}

\section{Gerry Ferguson}

Volume 21, numéro 2, 1988

Les soins de santé mentale en milieu carcéral

URI : https://id.erudit.org/iderudit/017263ar

DOI : https://doi.org/10.7202/017263ar

Aller au sommaire du numéro

Éditeur(s)

Les Presses de l'Université de Montréal

ISSN

0316-0041 (imprimé)

1492-1367 (numérique)

Découvrir la revue

Citer cet article

Ferguson, G. (1988). Le droit aux soins de santé mentale en milieu carcéral. Criminologie, 21(2), 13-25. https://doi.org/10.7202/017263ar

\section{Résumé de l'article}

Laws concerning prisoners and the psychiatric treatment of prisoners are neither clear, comprehensive nor readily accessible to those affected. Several factors which contribute to this state of «lawlessness» are examined. While the enacte-ment of the Canadian Charter of Rights and Freedoms has probably increased prisoners' rights to treatment, this right has not as yet been tested before the courts, nor has it's meaning been clearly defined. Prisoners' rights to refuse treatment are discussed, as well as the differing criteria for involuntary treatment laid out by the provincial mental health acts. Finally, the legal liability of health care professionals who treat, or fail to treat, accused or convicted offenders is addressed. 


\section{LE DROIT AUX SOINS DE SANTÉ MENTALE EN MILIEU CARCÉRAL Gerry Ferguson*}

Laws concerning prisoners and the psychiatric treatment of prisoners are neither clear, comprehensive nor readily accessible to those affected. Several factors which contribute to this state of "lawlessness" are examined. While the enactement of the Canadian Charter of Rights and Freedoms has probably increased prisoners' rights to treatment, this right has not as yet been tested before the courts, nor has it's meaning been clearly defined. Prisoners' rights to refuse treatment are discussed, as well as the differing criteria for involuntary treatment laid out by the provincial mental health acts. Finally, the legal liability of health care professionals who treat, or fail to treat, accused or convicted offenders is addressed.

Les lois sont décrétées afin de refléter le légitime intérêt que la société porte à la réglementation du comportement humain. Les lois fournissent une direction à suivre pour résoudre les revendications des réclamations résultant des conflits entre les citoyens. Pour atteindre leur but, les lois (et les droits et devoirs s'y rattachant) doivent être claires, compréhensibles et accessibles aux personnes concernées. Malheureusement, les lois concernant les détenus et le traitement psychiatrique des détenus ne sont pas, en général, claires, ni compréhensibles ni accessibles.

Plusieurs facteurs contribuent à cet état de vide juridique. Les droits des détenus (on entend rarement parler de leurs devoirs) occupent bien peu de place dans les affaires quotidiennes des législatures et des tribunaux. Le grand public et les législateurs se soucient fort peu du fait que les lois sur les prisons et les prisonniers soient incomplètes et inadéquates. C'est ainsi que ces lois incomplètes et inadéquates demeurent inchangées par nos législateurs. Et, comme l'accès aux tribunaux exige de l'argent (les procès coûtent cher) et que l'aide juridique ne s'étend généralement pas au domaine carcéral, cela signifie que les questions relevant du droit correctionnel se rendent rarement jusqu'aux tribunaux pour être interprétées et clarifiées.

Dans les cas où des causes ont été entendues par les tribunaux, les décisions prises sont habituellement peu sensibles aux droits des déte-

* Gerry Ferguson, professeur de droit et adjoint au doyen, faculté de droit, Université de Victoria, C.P. 2400 Victoria, Colombie-Britannique, V8W 3 H7. 
nus et souvent insuffisamment fondées. On dispose rarement de l'argent voulu pour porter la cause en appel devant un tribunal supérieur. Sans les jugements de la cour d'appel, les lois qui réglementent les affaires carcérales demeurent peu claires. La langue d'une loi est rarement absolue; jusqu'à ce qu'une cour d'appel ait statué sur le sens d'une loi, il est dangereux de se prononcer de façon dogmatique sur ce qu'elle est.

Le fait que l'autorité législative dans ce domaine soit partagée entre les gouvernements fédéral et provinciaux rend d'autant plus difficile une tentative pour résumer la loi gouvernant le traitement psychiatrique des détenus au Canada. Le gouvemement fédéral a l'autorité pour sanctionner des lois concernant l'administration des pénitenciers (réservés aux détenus purgeant une sentence de 2 ans ou plus) alors que chaque gouvernement provincial a l'autorité de légiférer au sujet des prisons et des institutions correctionnelles (pour les détenus purgeant des sentences de moins de 2 ans). De plus, la médecine, la psychiatrie et les hôpitaux psychiatriques sont de juridiction provinciale. Dans cet article, j'essaierai d'établir la distinction entre les lois affectant les détenus fédéraux et celles qui affectent les détenus provinciaux. Comme il existe dix différentes lois provinciales en vigueur, il convient d'être prudent et de ne pas prendre pour acquis que la position légale dans une province est la même dans toutes les autres. Ceci vaut surtout pour le Québec qui a un «système de droit civil» alors que les autres provinces ont un système de Common Law.

Pour compliquer encore les choses, la Charte canadienne des droits et libertés (1982) a ajouté une nouvelle couche d'incertitude à la loi concernant le traitement psychiatrique des détenus. Il est fort possible que la Charte ait augmenté substantiellement les droits des prisonniers soumis au traitement, mais personne ne le saura de façon certaine jusqu'à ce qu'un ou plusieurs d'entre eux aient trouvé assez d'argent (100000 \$ au moins) pour porter leurs plaintes devant la Cour suprême du Canada.

\section{LA CHARTE CANADIENNE DES DROITS ET LIBERTÉS}

À la manière des dispositifs de sécurité dans les aéroports, la Charte agit comme un mécanisme de protection à travers lequel toutes les autres lois et activités de l'état (fédérales ou provinciales) doivent passer san's encombre. L'article 52 de la Constitution canadienne établit que la Charte est la loi suprême du Canada et que toute autre loi (fédérale ou provinciale) qui entrerait en contradiction avec les droits et 
libertés prévus par la Charte est sans effet dans les limites de cette incompatibilité. Ce n'est que récemment que les causes relatives à la Charte ont commencé à parvenir devant la Cour suprême du Canada et, dans quelques cas spectaculaires, la Cour suprême a invalidé des lois existantes comme certaines dispositions relatives au meurtrel et à l'avortement ${ }^{2}$ en s'appuyant sur le fait qu'elles violaient certains droits de la Charte. Mais aucune de ces causes en Cour suprême n'avait à voir avec les droits des détenus et, par conséquent, il est difficile de prédire, avec quelque degré de certitude, de quelle manière la Charte affectera les droits des détenus en général et plus particulièrement leur droit au traitement.

Il y a un certain nombre de droits et libertés dans la Charte qu'il convient de relever ici :

1) L'article 2(b) de la Charte garantit que «chacun a droit à liberté de pensée, de croyance, d'opinion et d'expression». On peut en déduire qu'un traitement imposé par la force (non consenti) viole la liberté de pensée, de croyance et d'opinion de la personne (particulièrement s'il est appliqué à un patient-détenu en possession de ses facultés mentales). Aux États-Unis, dans la cause Kaimowitz ${ }^{3}$, on a utilisé avec succès un tel argument pour empêcher une opération de psychochirurgie chez un patient détenu contre son gré, alors que lui-même en faisait la demande.

Il ne fait aucun doute que la nature importune et irréversible du procédé de même que son efficacité controversée ont compté pour beaucoup dans la décision du tribunal.

2) L'article 7 de la Charte statue que «chacun a le droit à la vie, à la liberté et à la sécurité de sa personne; il ne peut être porté atteinte à ce droit qu'en conformité avec les principes de justice fondamentale». Cet article peut servir à démontrer que les patients-détenus ont le droit de refuser le traitement et, qu'à l'inverse, ils ont le droit au traitement ${ }^{4}$. Dans les cas où on peut imposer ou refuser le traitement, le patientdétenu a au moins le droit que les décisions au sujet de son traitement

1. Vaillancourt c. La Reine, Cour suprême du Canada, le 3 décembre, 1987.

2. Morgentaler c. La Reine, Cour suprême du Canada, le 28 janvier, 1988.

3. Kaimowitz c. Department of Mental Health, résumé dans: 42 U.S.L.W. 2063, excerpted in 2 Prison Law Reporter (1973).

4. Voir, par exemple, Collin c. Lussier, infra, note 10 et Peterson c. Minister of Health, Ontario Supreme Court, le 23 décembre, 1983, où le juge établit que:

My inclination is to assume that there occurs an attack upon the security of a contrary to his or her wishes to suffer an intake or the application of medication or 
soient prises en accord avec les principes de justice fondamentale qui comprennent le droit d'être entendu et représenté si nécessaire et le droit qu'une personne impartiale ou un comité décident de son cas's.

3) L'article 12 de la Charte établit que «chacun a droit à la protection contre tous traitement ou peine cruels et inusités». Encore une fois, cet article peut servir à prouver que des conséquences sérieuses découlant du non-traitement constituent un «traitement ou une peine cruels et inusités» et, qu'en contrepartie, l'imposition de certains types de traitements comme la psychochirurgie, les électrochocs ou certaines formes rigoureuses de renforcement négatif dans les programmes de modification du comportement constituent aussi des traitements cruels et inusités 6 .

4) L'article 15 de la Charte stipule que «la loi ne fait exception de personne et s'applique également à tous, et tous ont droit à la même protection et au même bénéfice de la loi, indépendamment de toute discrimination, notamment fondée sur la race, la religion, le sexe, l'âge ou les déficiences mentales ou physiques». Cet article peut servir à prouver que les détenus souffrant de déficiences mentales ont droit à la même qualité de services et aux mêmes protections légales que les personnes qui ne sont pas institutionnalisées.

5) Les restrictions de la Charte. Les droits et libertés énumérés plus haut ne sont pas absolus. L'article 1 de la Charte établit que les droits et libertés dans la Charte seront soumis à «des limites qui soient raisonnables et dont la justification puisse se démontrer dans le cadre d'une société libre et démocratique». Ainsi, même si les articles 2(b), 7 , 12 et 15, garantissent un droit au traitement ou un droit de refuser le traitement en général, ces droits peuvent être limités dans des cas précis, comme ceux des détenus par exemple, ou ceux des personnes ne jouissant pas de leurs facultés mentales, pourvu que les «limites soient

of a foreign substance ostensibly for the purpose of maintaining or improving his or her health. While there may well arise in certain circumstances a competing right to medical treatment as part of the obligation contained in and imposed by and pursuant to the same section 7 of ensuring the liberty and security of the person, I hold for the purpose of this motion at least, and my view on this matter accords with the general approach which I have espoused in other cases, that any encroachment upon the liberty and security of the person must be justified under section 1.

5. Voir, par exemple, The Queen c. Chester, Ontario Supreme Court, 1984.

6. Voir, M. Schiffer, Psychiatry Behind Bars (Toronto, Butterworths, 1982); Harvey Savage and Carla McKague, Mental Health Law in Canada (Toronto, Butterworths, 1987); J.L. Baudoin, «Le droit de refuser d'être traité», in Justice Beyond Orwell (R.S. Abella et M.L. Rothman, édit.) (Yvon Blais Inc., Montréal, 1985). 
raisonnables dans une société libre et démocratique». Ce qu'un tribunal considère «raisonnable» dépendra dans une large mesure de l'attitude générale que les tribunaux (et la société) adopteront vis-à-vis des détenus et des patients psychiatriques. À travers l'histoire, les tribunaux (et la société) se sont permis des ingérences massives dans les droits des détenus et des patients psychiatriques, en qualifiant ces intrusions de raisonnables ou de nécessaires au bien public ou au bien des patientsdétenus eux-mêmes. L'avenir dira si de telles ingérences continueront à être permises au nom de la clause de «limite raisonnable» de l'article l de la Charte.

\section{LE DROIT AU TRAITEMENT}

Les détenus ont-ils à l'heure actuelle, un droit légal au traitement, au Canada? Malheureusement, la réponse à cette question demeure incertaine. Les garanties de la Charte portant sur le droit à la vie, à la liberté et à la sécurité de la personne et l'interdiction qu'elle émet contre les traitements et les peines cruels et inusités sont suffisamment larges pour inclure un droit légal au traitement pour les détenus?

Au cours des 25 dernières années, les autorités correctionnelles ont graduellement élargi le champ des droits et des privilèges des détenus canadiens. Cependant, la plupart des programmes et des activités dans les prisons canadiennes sont considérés officiellement comme des privilèges et non comme des droits, «et peuvent être arbitrairement retirés à un individu ou à tous, pour des raisons de sécurité, de politique administrative ou de commodité ou bien à cause de l'inconduite d'une personne ou d'un groupe ${ }^{{ }^{\prime}}$. Avant l'avènement de la Charte, les tribunaux canadiens n'examinaient normalement pas le traitement des détenus dans les institutions pénales. Depuis que les services et les programmes psychiatriques et psychologíques sont à court de ressources dans les prisons, les détenus qui s'étaient vu refuser l'accès à des programmes nécessaires ou utiles, n'avaient pas de recours légal apparent.

7. Savage and McKague, op. cit., ch. 5.

8. H.R.S. Ryan, «The Impact of the Canadian Charter of Rights and Freedoms on the Canadian Correctional System», in (1983) 1 Canadian Human Rights Yearbook 99. Ryan résume ainsi le manque de droits juridiques dans les prisons canadiennes (p. 111): «Inmates may be placed in and removed from any degree of security and segregation and any institution. Within an institution they may be placed in or denied any programme by decisions in which they are not entitled to take part and of which they are not, as a matter of law, entitle to notice in advance. In practice, if being considered for transfer to a special handling unit, they may be notified but will not be given a voice in the decision. They may grieve about these decisions but are not likely to gain much through grievances» Voir aussi L. Vandervort, "Legal Aspects of the Medical Treatment of Penitentiary Inmates» (1976-1977) 3, Queen's Law J. 368. 
En ce qui concerne les détenus fédéraux, la Loi sur les pénitenciers ne contient aucune disposition relative aux droits au traitement. Cependant, selon cette Loi, le Commissaire des pénitenciers a le pouvoir d'établir des règles qu'on appelle les Directives du commissaire.

Les Directives du commissaire, actuellement en vigueur, imposent expressément au service correctionnel le devoir de fournir aux détenus des services en santé mentale. Mais ces Directives, contrairement aux lois et règlements, ne confèrent pas de droits juridiques aux détenus. Normalement, elles sont considérées comme des politiques administratives internes que les tribunaux n'appliqueraient pas 9 . Cependant, les règlements du service des pénitenciers sont des lois. L'article 16 dit expressément que les soins médicaux doivent être fournis en accord avec les Directives. Depuis que l'article 16 appuie spécifiquement les Directives en ce qui concerne les soins médicaux, on peut soutenir que ces directives ont maintenant le statut de loi, du moins est-ce ce que la cour a soutenu dans la cause Collin c. Lussier ${ }^{10}$.

Dans l'affaire Collin c. Lussier, la Cour fédérale a aussi déclaré qu'en vertu de l'article 7 de la Charte canadienne, un détenu a le droit aux soins de santé comme faisant partie du droit à la sécurité de sa personne. Dans cette cause, la cour a soutenu que le transfèrement du détenu dans une autre prison était inconstitutionnel parce que, dans la nouvelle institution, les services de soins médicaux de nuit étaient insuffisants pour une personne qui, comme le détenu, souffrait d'attaques coronariennes. Il reste à savoir si un tribunal canadien appliquerait le même principe au cas où des traitements psychiatriques ou psychologiques seraient exigés. La cause américaine Bowring c. Goodwin ${ }^{11}$ démontre un haut niveau d'exigences quant aux conditions requises pour le droit au traitement - le détenu doit être atteint d'une maladie ou d'une blessure graves qu'on peut guérir ou soulager de façon notoire et pour lesquelles tout retard ou absence de soins causerait un tort ou un préjudice considérable au détenu.

En règle générale, les tribunaux canadiens n'ont été réceptifs que dans les cas de détresse extrême. Ainsi, on a considéré comme un traitement cruel et inusité, les conditions de détention «dans le trou», au pénitencier de la Colombie-Britannique où sévissait une privation extrême de lumière, d'air frais, d'exercice, etc. qui sont des besoins

9. (1978) 1 R.C.S. 118.

10. (1983) 1 C.F. 218 , décision renversée pour d'autres motifs.

11. (1977), 551 F. 244. 
humains fondamentaux ${ }^{12}$. Cependant, dans trois autres cas au Canada, les prisonniers n'ont pas réussi à faire admettre comme un traitement et une peine cruels et inusités, des conditions de détention moins excessives comme le surpeuplement, la double occupation et le manque d'exercice en plein air's.

En ce qui concerne le droit au traitement des détenus provinciaux, on doit consulter les lois et les règlements de chacune des provinces. Par exemple, en Colombie-Britannique, les Lois et règlements des établissements de détention exigent que les agents correctionnels qui soupçonnent qu'un détenu souffre d'une maladie physique ou mentale sérieuse rapportent la chose à l'officier responsable ou au médecin de l'institution, le plus tôt qu'il leur est raisonnablement possible de le faire ${ }^{14}$. L'officier médical a «la responsabilité de satisfaire aux besoins concernant la santé physique et mentale des détenus»15.

Quand un détenu requiert un «traitement médical dans un service de santé mentale provincial, l'officier médical devra en aviser le directeur et l'aider à organiser son transfèrement»16. Au Québec, dans les Lois et règlements concernant les établissements de détention, la section $X$, intitulée soins de santé, se lit ainsi ${ }^{17}$ :

19. Une personne incarcérée doit pouvoir recevoir les soins de santé que requiert son état.

20. Une personne incarcérée dont l'état le requiert doit être transférée dans un centre hospitalier.

21. Une personne incarcérée ne peut être soumise à des expériences médicales et scientifiques pouvant porter atteinte à son intégrité physique ou mentale.

22. Un professionnel de la santé de l'établissement doit présenter un rapport à l'administrateur chaque fois qu'il estime que la santé physique ou mentale d'une personne incarcérée a été ou sera affectée par les conditions de détention qui lui sont imposées ou par leur prolongation.

12. McCann c. La Reine (1976), 29 C.C.C. (2e) 337 (Cour fédérale, T.D.). Voir aussi M. Jackson, Prisoners of Jsolation : Solitary Confinement in Canada (U. of Toronto Press : 1983).

13. Soenen c. Director of Edmonton Remand Centre, (1983), 6 Charter of Rights Reporter 364 (Alta. Q.B.) ; Collin c. Kaplan (1982), 2 Charter of Rights Reporter 352 (Cour fédérale, T.D.); Piche c. The Solicitor General of Canada Federal Court, Trial Division, 2 novembre 1984. Voir aussi Paul Russell, «Curel and Unusual Treatment or Punishment: The Use of Section 12 in Prison Litigation» (1985), 43 Univ. Toronto Faculty Law Review 185.

14. Correction Centre Rules and Regulations, B.C. Regulations, 284/78, s. 12.

15. Ibid., s. 51 (1).

16. Ibid., s. 49.

17. Statuts refondus du Québec, 1981, chap. 26, a 1. 
Bien que ces règles semblent claires et nettes, il n'existe aucun cas devant les tribunaux qui démontre jusqu'où iront les tribunaux pour forcer les gouvernements provinciaux et fédéral à fournir les traitements psychiatriques et psychologiques nécessaires. Le contenu du droit au traitement va forcément être limité par les moyens dont disposera la société pour fournir de tels services. Nous savions qu'environ 20 à $25 \%$ de la population des pénitenciers fédéraux souffre de sérieux troubles psychiatriques, psychologiques ou de comportement ${ }^{18}$. Cela relève de notre expertise et de nos ressources actuelles de répondre à ce besoin. Le rapport Carson ${ }^{19}$ et le rapport MacGuigan ${ }^{20}$ indiquent que le public est prêt à ce que le traitement de tels désordres fasse partie des services de santé mentale qui devraient être accessibles aux détenus et le numéro 11 de la revue Operationa ${ }^{21}$ révèle une préoccupation réelle du personnel et de l'administration pour que de tels services soient offerts.

La priorité qui a été accordée au cours des quinze dernières années aux désordres psychiatriques graves dans les établissements fédéraux est louable mais on doit trouver aussi les ressources nécessaires pour faire face aux sérieux désordres psychologiques et aux troubles de comportement.

\section{LE DROIT DE REFUSER LE TRAITEMENT}

En général, le droit canadien reconnaît à l'adulte en possession de ses facultés mentales le droit de refuser un traitement médical même si la conséquence doit en être la mort ${ }^{22}$. Par exemple, un témoin de

18. J.S. Wormith, et M. Borzecki, les Maladies mentales dans le système de justice pénale solliciteur général, Ottawa, $1985 \mathrm{p} .56: \mathrm{Si}$ on les considère globalement, les études appuient l'affirmation selon laquelle, dans les populations carcérales générales, on peut estimer que $5 \%$ des détenus souffrent d'une maladie mentale grave qui nécessite des soins spécialisés et $20 \%$, de troubles mentaux et de la personnalité moins sérieux qui devraient être traités.

19. Rapport du comité consultatif chargé par le solliciteur général du Canada d'étudier la gestion des établissements correctionnels (solliciteur général, Ottawa, 1984) (Rapport Carson).

20. Rapport au Parlement du sous-comité sur le régime d'institutions pénitentiaires au Canada (Ottawa, Approvisionnements et services, 1977) (Rapport MacGuigan).

21. Operational and Resource Management Review : Review Number 11 : Review of Mental Health Service (solliciteur général, Ottawa, 1985).

22. Voir note 6, Supra. Dans l'affaire Schloendorf c. N.Y. Hospital (1914), 211 N.Y. 127 , à la p. 129,105 N.E. 92, à la p. 93, le juge Cardozo déclare : Every human being of adult years and sound mind has a right to determine what shall be done with his or her own body. Voir aussi la Commission de réforme du droit du Canada, Document de travail 43, les Techniques de modification du comportement et le droit pénal (Ottawa, Approvisionnements et services, 1980) pp. 41-47; M. Goehnauer, «Refusal of Medical Treatment: Taking Respect for the Person Seriously» (1982), 2 Can. J. Law \& Society 121; A. Macklin, «Bound to Freedom: The Ulysses Contract and the Psychiatric Will» (1987), 45 University of Toronto Faculty Law Review 37. 
Jéhovah adulte peut refuser une transfusion sanguine qui lui sauverait la vie. Quand un adulte n'est pas jugé apte à prendre les décisions à son propre sujet ou au sujet de son traitement, de telles décisions reviennent à un membre de sa famille, à son tuteur ou à un tribunal. Le droit de refuser un traitement est basé sur le respect de la société pour l'autonomie de l'individu et sa vie privée.

On retrouve ces valeurs dans la Charte canadienne des droits et libertés. La loi protège le droit du citoyen à demeurer en paix. Les ingérences non consenties constituent une offense criminelle ou un tort civil. Ainsi, une intervention médicale ou un traitement sans le consentement valide du patient constitue une agression ${ }^{23}$.

Ces principes généraux s'appliquent-ils aussi aux détenus? On admet généralement que le prisonnier adulte qui n'a pas été déclaré légalement privé de ses facultés mentales ou n'a pas été confié à un hôpital psychiatrique en vertu du code civil, possède le même droit de refuser le traitement que tout citoyen ordinaire ${ }^{24}$. Ainsi, un détenu peut refuser le traitement psychiatrique ou psychologique, même si ce traitement peut éliminer ou réduire ses tendances criminelles ${ }^{25}$. Il peut y avoir une exception au droit du détenu à refuser le traitement si ce refus de traitement menace sa vie. Par exemple, il n'est pas certain que les dirigeants d'une prison aient le devoir légal de nourrir de force un détenu qui va mourir à la suite d'une grève de la faim ${ }^{26}$.

23. G. Sharpe, The Law and Medicine in Canada (Toronto, Butterworths, $2^{\mathrm{e}}$ éd. 1987), ch. 4 ; E. Picard, Legal Liability of Doctors and Hospitals in Canada (Toronto, Carswell, $2^{\mathrm{e}}$ éd. 1984), ch. 3 ; A. Linden, Canadian Tort Law (Toronto, Butterworths, $3^{\mathrm{e}}$ éd., 1982), pp. 57-66.

24. Dans l'affaire Regina c. Berrie, (1975) 30 C.R.N.S. 145, une cour canadienne a reconnu un certain nombre d'agents de correction coupables d'assaut pour avoir utilisé une force non permise, pour raser un détenu. La cour a soutenu qu'en l'absence d'urgence ou de nécessité ils auraient dû avoir recours au processus disciplinaire prévu afin de faire respecter les règlements de la prison au sujet du port de la barbe, plutôt que d'utiliser la force. La Cour suprême du Canada dans la cause Solosky c. La Reine (1979), 50 C.C.C. $\left(2^{\mathrm{e}}\right) 495$ a soutenu que les détenus ont droit, en général, à profiter également de la protection et des avantages de la loi.

25. De tels refus sont fréquents. Voir J.S. Wormith, «Survey of incarcerated sexual offenders» (1983) 25, Can. J. of Criminology, p. 379. L'auteur affirme que les délinquants sexuels forment à peu près $10 \%$ de la population carcérale et qu'approximativement $70 \%$ d'entre eux ne sont pas intéressés à participer à un programme de traitement dans le milieu carcéral qu'ils occupent.

26. Il y a quelque incertitude à savoir si un prisonnier ou une personne détenue pour un autre motif par l'état a le droit de refuser un traitemtn. Une cause en Angleterre, Leigh c. Gladstone (1909), 26 T.L.R. 139 (K.B.), a soutenu que les officiers de la prison avaient le devoir légal de nourrir de force une détenue et n'étaient pas coupables d'assaut en le faisant. Dans la cause Attorney-General for British Columbia c. Astaforoff (1983), 6 C.C.C. (3e) 503 (C.A.C.B) conf., 6 C.C.C. (3e) 498 (C.S.C.B), le gouvernement fédéral 
En dehors des situations où sa vie est menacée, la pratique courante dans les prisons canadiennes est de reconnaître au détenu le droit de refuser un traitement et d'exiger qu'il ou elle donne son consentement éclairé à tout traitement qui lui est imposé27.

Les centres psychiatriques régionaux qui font partie du réseau fédéral des établissements de détention fonctionnent en prenant pour acquis qu'un consentement éclairé est nécessaire à l'administration de tout programme de traitement. Quant au consentement, les exigences normales sont qu'il soit à la fois éclairé et volontaire. Un consentement éclairé exige que le patient-détenu ait été informé de façon adéquate des risques du traitement. En pratique, ceci est rarement fait adéquatement ${ }^{28}$.

Les exigences pour un consentement volontaire sont plus difficiles à obtenir en milieu carcéral. La participation aux programmes de traitement peut être une condition nécessaire à une libération anticipée ou au transfèrement vers une institution à sécurité moins élevée et dont le régime comporte plus de privilèges. Certains maintiennent que le consentement est involontaire quand le traitement est offert aux détenus comme une condition de libération anticipée. Cette pratique est particulièrement gênante quand le traitement est fortement importun ou que les effets secondaires appréhendés sont considérables ou inconnus

a demandé à la Cour d'appel de la Colombie-Britannique d'émettre un ordre afin d'autoriser qu'on alimente de force Mary Astaforoff, une vieille dame Doukobor de 69 ans, qui avait reçu une sentence d'emprisonnement de 3 ans pour sa septième condamnation pour incendie criminel. La cour a refusé d'émettre un tel ordre car elle n'était pas certaine qu'il existait un tel devoir d'alimenter un personne de force. Elle laissa l'affaire entre les mains du gouvernement. Le gouvernement alla de l'avant et nourrit la dame de force de sa propre initiative.

Dans Solliciteur général du Canada c. Niemic, (1984) C.S. 426, 8 C.R.R. 382, la Cour supérieure du Québec ordonna un traitement médical nécessaire sur $\mathrm{N}$. Niemic, un citoyen polonais, à qui l'on avait refusé le statut de réfugié et qui était détenu en attendant d'être déporté. Dans une dernière tentative pour éviter la déportation, il avait avalé intentionnellement un long fil de fer qui aurait facilement pu lui perforer la gorge ou l'estomac s'il était déplacé. En refusant la chinurgie destinée à retirer le fil, il affirma qu'il préférait mourir au Canada que de retourner en Pologne. Malgré lui, la Cour émit un ordre pour autoriser la chinurgie. La cour considéra que les droits de $\mathrm{N}$. Niemic à l'autonomie et à l'autodétermination étaient soumis à diverses restrictions reconnues par la loi. On peut se demander si ces cas peuvent être généralisés au-delà de leurs faits particuliers. Voir D. Caswell, "Limitations in Canadian Law on the Right of a Prisoner to Refuse Medical Treatment» (1986), 2 J. Contemp. Health L. and Policy, 155 ; N. Somerville, «Refusal of Medical Treatment in Captive Circumstances» (1985), 65, Canadian Bar Review 59.

27. Dans les pénitenciers fédéraux, l'instruction 700-2-02 (1982-11-30) expose en détail les politiques à suivre pour l'obtention d'un consentement éclairé au traitement médical, psychiatrique ou dentaire.

28. Oak ridge: A Review and An Alternative (S.J. Hucker, Chairman) (Minister of Health, Ontario, 1966). 
comme dans le cas de la psychochirurgie ${ }^{29}$. Cependant, il est peu vraisemblable que le consentement d'un détenu à des traitements moins violents puisse s'avérer involontaire du simple fait que le traitement aurait pour résultat de rapprocher la date de sa libération conditionnelle.

La Commission de réforme du droit du Canada dans son document de travail 43 établit que la seule présence de difficultés liées au consentement du détenu n'est pas une raison suffisante pour conclure que les prisonniers ne peuvent jamais donner un consentement libre au traitement. La Commission croit que la solution repose dans le mécanisme utilisé pour s'assurer d'un consentement éclairé et libre. À ce sujet, la Commission dit :

Le traitement doit rester simplement accessible et ne doit pas être indirectement imposé. Il ne doit pas non plus être formellement relié à des faveurs, à des remises de peine ou à des privilèges. Le principe de la proportionnalité entre le bénéfice espéré et les risques encourus doit enfin être scrupuleusement respecté et l'unique considération demeurer la recherche du mieux être du détenu...

Il convient toutefois d'être prudent, de prendre des précautions supplémentaires pour s'assurer que le consentement donné soit parfaitement éclairé, d'éliminer le plus possible les facteurs de coercition et de renforcer les mécanismes assurant un consentement volontaire et éclairé 30 .

\section{LE TRANSFÈREMENT DANS DES SERVICES PROVINCIAUX DE SANTÉ MENTALE}

Quand un détenu est transféré dans un service provincial de santé mentale, les dispositions prévues dans la Loi sur la santé mentale s'appliquent. La législation en santé mentale varie d'une province à l'autre. Dans certains cas, elle est muette et dans d'autres cas, elle semble autoriser explicitement ou implicitement le traitement sans consentement ${ }^{31}$. Si un patient a été admis de son plein gré et qu'il est compétent mentalement, il ou elle a généralement le droit de refuser un traitement. Cependant, si un patient est confié contre son gré à l'hôpi-

29. Voir par exemple, Kaimowitz c. Department of Mental Health, supra note 3, où, en dépit de l'apparent consentement du détenu et de l'approbation d'un comité scientifique de révision, le tribunal a soutenu que l'opération de psychochirurgie proposée était inconstitutionnelle.

30. Commission de réforme du droit du Canada, document de travail 43 , les Techniques de modification du comportement et le droit pénal, pp. 37-49.

31. Ibid., pp. 22-23. 
tal, il ou elle perd habituellement le droit au traitement. Savage et McKague résument ainsi la situation :

Dans plusieurs juridictions, le simple fait d'y être envoyé autorise l'hôpital à appliquer le traitement; c'est le cas en Alberta, en Colombie-Britannique, au Manitoba, au Nouveau-Brunswick, à Terre-Neuve, dans les Territoires du Nord-Ouest et en Saskatchewan.

Il n'est fait aucune mention de l'autorisation de traiter les patients involontaires sans leur consentement dans les lois de l'Île-duPrince-Édouard et du Yukon et on présume que le droit au refus reconnu en Common Law s'applique toujours, au moins pour les patients en possession de leurs facultés mentales.

En Nouvelle-Écosse, aucun traitement ne peut être donné sans le consentement du patient, s'il jouit de ses facultés mentales; dans le cas contraire, le consentement doit venir de son tuteur, de son épouse, de ses plus proches parents ou du curateur public.

En Ontario, un patient apte à prendre une décision peut refuser; un patient incapable de consentir personnellement, peut être traité avec le consentement de son plus proche parent ou, si celui-ci ne s'en trouve aucun, sur l'ordre d'un comité régional de révision. Cependant, le refus d'un patient ou d'un de ses proches peut être outrepassé par un comité régional de révision si celui-ci est persuadé que le traitement proposé est susceptible d'aider substantiellement le patient et que ce dernier ne progresserait vraisemblablement pas sans lui. La décision du comité peut maintenant être portée en appel devant une cour du district.

Le Québec a la législation la plus étrange en ce domaine. Dans cette province, une personne a le droit de refuser le traitement si elle est jugée apte à gérer ses affaires financières. Autrement, les biens du patient sont placés sous le contrôle du curateur public à qui, avec ce contrôle financier, revient aussi le droit de prendre les décisions sur le traitement, au nom du patient (Si le patient a déjà un curateur privé, ce dernier est autorisé à donner son consentement.) En pratique, la signature du curateur public n'est requise que pour les interventions qui nécessitent une anesthésie comme la chirurgie et les électrochocs. Les autres interventions sont laissées au bon jugement des administrateurs hospitaliers 32 .

La Colombie-Britannique est la seule province qui possède, dans la loi sur la santé mentale, une disposition expresse autorisant l'application (non consentie) d'un traitement à des personnes transférées d'une prison dans un hôpital psychiatrique ${ }^{33}$. Qu'on traite une personne transférée d'une prison dans un hôpital psychiatrique comme un patient

32. Savage and McKague, supra note 6, pp. 107-108.

33. British Columbia Mental Health Act, R.S.B.C. 1979, c. 256 comme amende, art. 8 (1) (a). 
consentant officieusement ou comme un patient qui s'oppose au traitement dépendra ou bien des procédures légales employées pour le transfèrement (c'est-à-dire si elle a été hospitalisée en vertu du code civil, ou non) ou bien si le patient-détenu est apte à consentir au traitement ou non.

Quand une personne est envoyée dans un hôpital psychiatrique avec un mandat du lieutenant-gouverneur pour inaptitude ou aliénation mentale vis-à-vis d'une infraction criminelle, le code criminel ne dit pas si cette personne peut être traitée sans son consentement ou non. Au Québec, le cas de l'Institut Philippe-Pinel c. Dion ${ }^{34}$ stipule qu'une personne détenue en vertu d'un mandat du lieutenant-gouverneur peut être traitée sans son consentement et contre sa volonté, mais seulement après que l'hôpital ait obtenu le consentement de la Cour. L'avenir dira si cette cause sera appliquée dans les autres provinces.

\section{LA RESPONSABILITÉ LÉGALE DU PERSONNEL}

Les professionnels de la santé sont vulnérables, du point de vue de leur responsablité légale, pour avoir ou bien fait trop ou bien fait trop peu dans le traitement des patients-détenus. La ligne de conduite la plus sûre pour eux est de ne traiter les patients-prisonniers qu'après qu'ils aient donné leur consentement éclairé au traitement. Si un patientdétenu refuse le traitement, ce refus doit être respecté à moins que la personne ne paraisse inapte à prendre cette décision. Si tel est le cas, il faut porter la chose à l'attention de la direction de l'institution afin d'obtenir une confirmation légalement valide de l'inaptitude mentale de la personne à consentir au traitement. Dans de pareils cas, on peut obtenir un consentement par procuration.

Il est malheureux que la loi soit si peu claire en ce qui regarde le traitement psychiatrique des détenus et des patients psychiatriques. Les professionnels de la santé devraient exiger que les gouvernements fédéral et provinciaux édictent des lois qui expliciteraient clairement le droit au traitement et le droit de refuser le traitement. 